\title{
TU/e EN⿴HONE

\section{Fabrication, detection, and operation of a three-dimensional nanomagnetic conduit}

\section{Citation for published version (APA):}

Sanz-Hernández, D., Hamans, R. F., Liao, J. W., Welbourne, A., Lavrijsen, R., \& Fernández-Pacheco, A. (2017). Fabrication, detection, and operation of a three-dimensional nanomagnetic conduit. ACS Nano, 11(11), 1106611073. https://doi.org/10.1021/acsnano.7b05105

\section{Document license:}

CC BY

DOI:

10.1021/acsnano.7b05105

Document status and date:

Published: 28/11/2017

\section{Document Version:}

Publisher's PDF, also known as Version of Record (includes final page, issue and volume numbers)

\section{Please check the document version of this publication:}

- A submitted manuscript is the version of the article upon submission and before peer-review. There can be important differences between the submitted version and the official published version of record. People interested in the research are advised to contact the author for the final version of the publication, or visit the $\mathrm{DOI}$ to the publisher's website.

- The final author version and the galley proof are versions of the publication after peer review.

- The final published version features the final layout of the paper including the volume, issue and page numbers.

Link to publication

\section{General rights}

Copyright and moral rights for the publications made accessible in the public portal are retained by the authors and/or other copyright owners and it is a condition of accessing publications that users recognise and abide by the legal requirements associated with these rights.

- Users may download and print one copy of any publication from the public portal for the purpose of private study or research.

- You may not further distribute the material or use it for any profit-making activity or commercial gain

- You may freely distribute the URL identifying the publication in the public portal.

If the publication is distributed under the terms of Article $25 \mathrm{fa}$ of the Dutch Copyright Act, indicated by the "Taverne" license above, please follow below link for the End User Agreement:

www.tue.nl/taverne

Take down policy

If you believe that this document breaches copyright please contact us at:

openaccess@tue.nl

providing details and we will investigate your claim. 


\title{
Fabrication, Detection, and Operation of a Three-Dimensional Nanomagnetic Conduit
}

\author{
Dédalo Sanz-Hernández, ${ }^{\dagger}$ Ruben F. Hamans, ${ }^{\ddagger}$ Jung-Wei Liao, ${ }^{\dagger}$ Alexander Welbourne, ${ }^{\dagger}$ \\ Reinoud Lavrijsen, ${ }^{\ddagger}$ and Amalio Fernández-Pacheco* ${ }^{* \dagger}$ \\ ${ }^{\dagger}$ Cavendish Laboratory, University of Cambridge, JJ Thomson Avenue, Cambridge CB3 OHE, United Kingdom \\ ${ }^{*}$ Department of Applied Physics, Eindhoven University of Technology, P.O. Box 513, 5600 MB Eindhoven, The Netherlands
}

\author{
Supporting Information
}

ABSTRACT: Three-dimensional (3D) nanomagnetic devices are attracting significant interest due to their potential for computing, sensing, and biological applications. However, their implementation faces great challenges regarding fabrication and characterization of 3D nanostructures. Here, we show a 3D nanomagnetic system created by $3 \mathrm{D}$ nanoprinting and physical vapor deposition, which acts as a conduit for domain walls. Domains formed at the substrate level are injected into a 3D nanowire, where they are controllably trapped using vectorial magnetic fields. A darkfield magneto-optical method for parallel, independent measurement of different regions in individual 3D nanostructures is also demonstrated. This work will facilitate the advanced study and exploitation of 3D nanomagnetic systems.

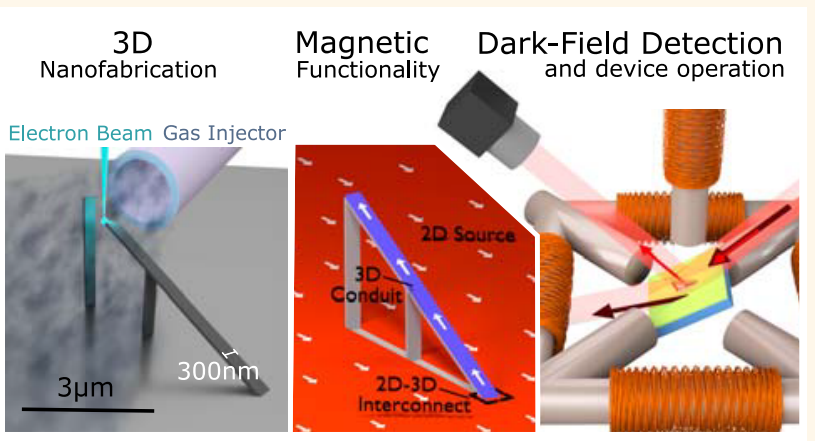

KEYWORDS: nanomagnetism, domain wall, magneto-optical Kerr effect, dark field, racetrack memory, 3D nanoprinting

M agnetic conduits based on nanowires (NWs) are one of the most advanced nanomagnetic systems ever realized. ${ }^{1-9}$ These devices exploit the domain wall (DW) conduit behavior, ${ }^{10}$ where DWs flow along NWs as a collection of separated objects, controlled by a wide range of mechanisms (magnetic fields, electrical/spin currents, spin waves, strain, etc.). In these systems, wires are not mere interconnectors but become the device itself.

Extensive knowledge has been acquired during recent years regarding the physics of DWs in NWs: phase diagrams, ${ }^{11,12}$ injection, motion and trapping, ${ }^{13,14}$ logic gates, ${ }^{1,15}$ mechanisms driving DW motion, ${ }^{16-18}$ dynamic properties $^{19,20}$ are some examples of the great progress achieved. DW conduits are now used in multiple fields, including spintronic circuits, ${ }^{1-3}$ automotive sensing, ${ }^{6}$ lab-on-a-chip applications, ${ }^{5,8,9}$ or for fundamental studies in complex nanomagnetic networks. ${ }^{7}$ However, although two-dimensional (2D) NW devices are now a reality, 3D NW devices are yet to be realized due to phenomenal patterning and characterization challenges. ${ }^{3,21}$ Until now, DW motion perpendicular to the substrate plane has been generally studied by means of out-of-plane NWs made by chemical methods and templates. ${ }^{22,23}$ This approach is nevertheless restricted to disconnected, vertical straight NWs and usually involves the release of the wires from the templates for subsequent characterization. Moreover, higher density of defects and rougher interfaces prevent smooth DW motion in comparison with their planar counterparts, ${ }^{23}$ where materials grown by physical methods are used. Alternative ways to create and probe high-quality magnetic NWs forming real 3D geometries are therefore essential for further progress in this area.

Here, we show a 3D nanomagnetic conduit created by a combination of direct-write nanolithography and standard physical vapor deposition. DWs are controllably injected from the substrate plane into a $3 \mathrm{D} \mathrm{NW}$ interconnected at an angle, where they move and become trapped at different locations under the application of external vectorial magnetic fields. A magneto-optical dark-field method which independently probes the substrate and the $3 \mathrm{D} \mathrm{NW}$ is employed to characterize the nanodevice. The methodology presented here can be extended to complex materials and geometries, enabling advanced studies of $3 \mathrm{D}$ nanomagnets.

\section{RESULTS}

3D Nanofabrication. The 3D magnetic conduit was created via a two-step lithography process: In step 1, a nonmagnetic scaffold is built using 3D nanoprinting via focused electron beam induced deposition ${ }^{24}$ (Figure 1a). In step 2, a

Received: July 19, 2017

Accepted: October 18, 2017

Published: October 26, 2017 

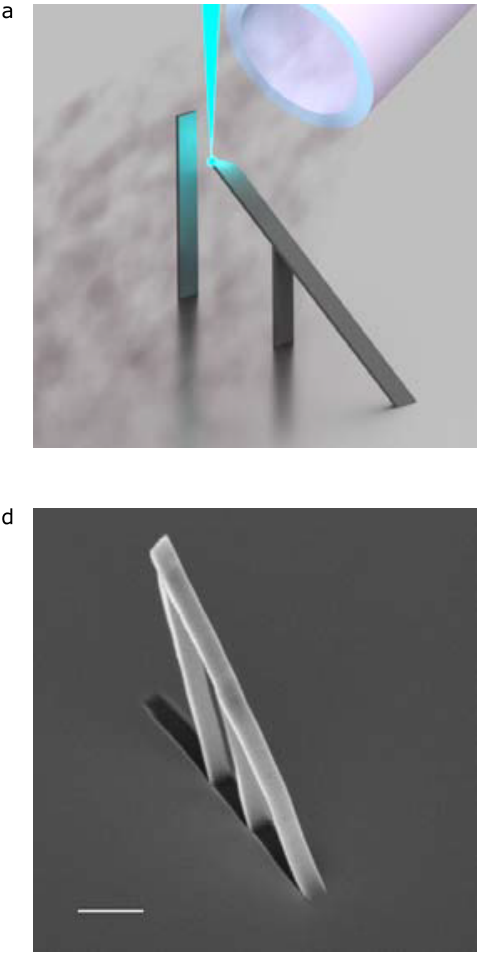
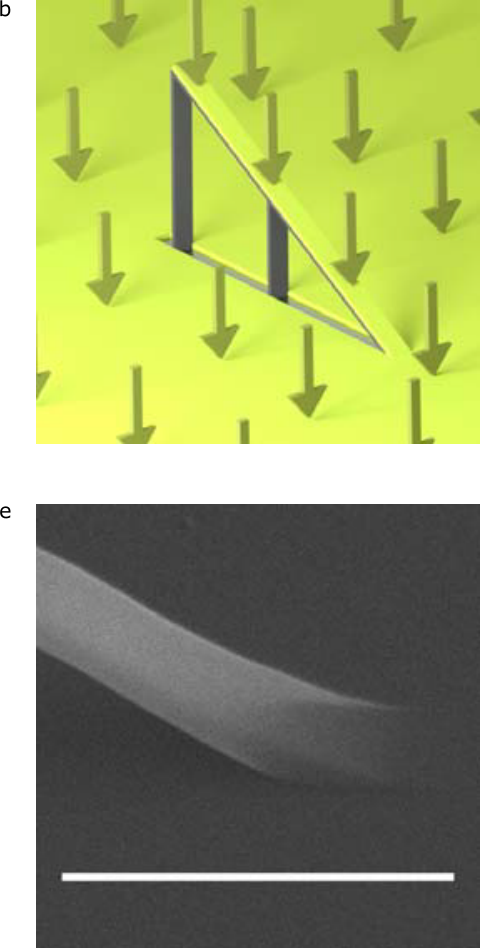
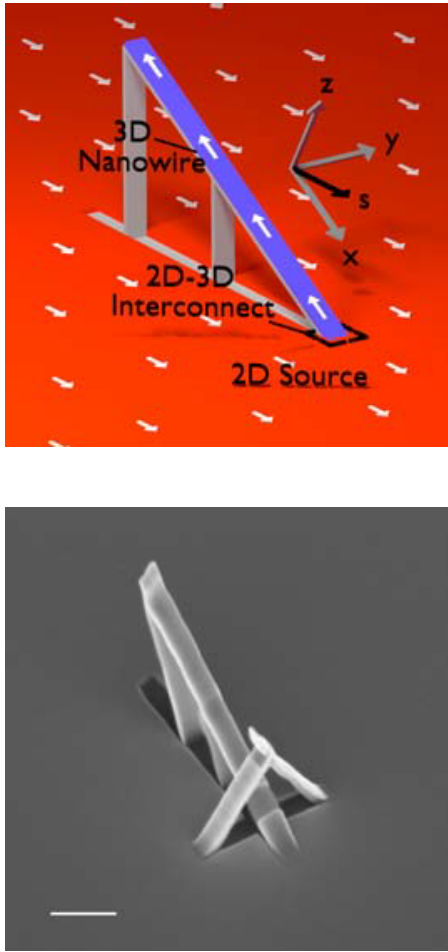

Figure 1. Fabrication of a 3D magnetic domain wall conduit. (a) 3D printing of a nonmagnetic scaffold using focused electron beam induced deposition. (b) 3D magnetic nanowire created by depositing a magnetic layer using thermal evaporation. (c) Schematic of the nanomagnetic system designed. The magnetic state of the $2 \mathrm{D}$ source (red) can be transferred into the 3D conduit (blue) via a 2D-to-3D interconnect. (d) SEM image of a nanomagnetic conduit after Permalloy evaporation $(50 \mathrm{~nm})$. The shadow created by the scaffold after evaporation is observed. (e) SEM image of the substrate-to-scaffold connection. (f) SEM image of a control nanostructure, disconnected from the 2D source by adding a nanobridge at its base, which shadows the growth of evaporated magnetic material on the area beneath it. Scale bars $1 \mu \mathrm{m}$.
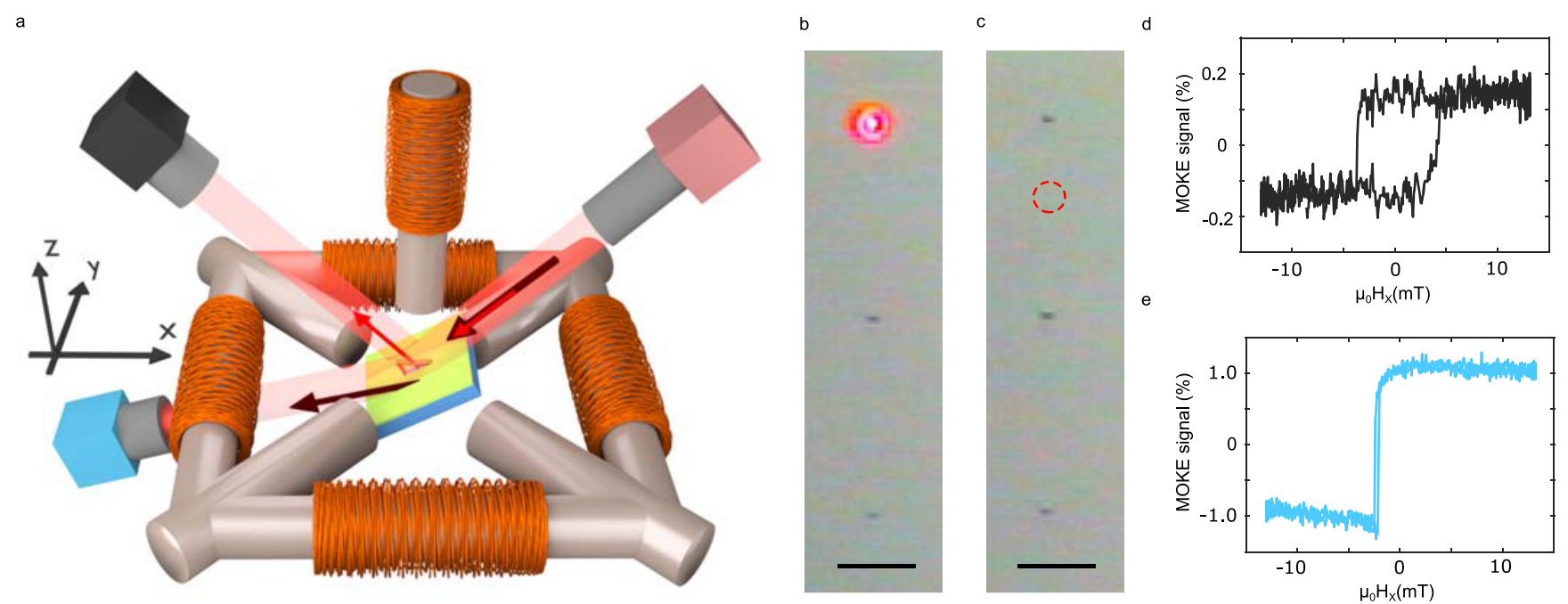

Figure 2. Magneto-optical detection. (a) Dark-field magneto-optical Kerr effect setup to detect independently a magnetic thin film and 3D NW under vectorial external magnetic fields. A single laser is directed at $45^{\circ}$ to the $x y$ plane, defined by the nanoramp. Most of the signal is reflected from the substrate into the blue detector (bright field). The contribution from the NW is detected by the black detector with no contribution from the film (dark field). The angle between the substrate and $x y$ planes is $33 \pm 3^{\circ}$, matching the NW inclination. (b) Optical microscopy image obtained with a CCD camera positioned at the same angle as the black detector. White light is sent to the sample at the appropriate angle. Three NWs are observed as black dots. The laser light is observed as reflected from a NW. (c) Sample is moved $20 \mu \mathrm{m}$ up, resulting in no laser light observed by the camera (laser position marked with dotted circle). (d) NW and (e) film hysteresis loops measured in parallel using this setup, upon application of a $14 \mathrm{mT}$ oscillating $x$-field and a constant $z$-field offset of $3.2 \mathrm{mT}$. Scale bars $20 \mu \mathrm{m}$.

magnetic thin film is deposited over the whole sample using thermal evaporation (Figure $1 \mathrm{~b}$ ). In this work, a nanoramp forming an angle with the substrate is used as the nonmagnetic scaffold. Two supporting pillars are added to ensure mechanical stability throughout the whole fabrication and characterization process. A curved interconnecting section is introduced 
between the ramp and the substrate to enable a smooth DW transmission from the $2 \mathrm{D}$ plane into the $3 \mathrm{D}$ NW. Permalloy is chosen as the evaporated magnetic material, due to its good DW conduit properties in $2 \mathrm{D}$ NWs. ${ }^{1,2}$ It also shows a magnetic response dominated by shape anisotropy, avoiding complex behaviors that would obscure a clear interpretation of results. A detailed account of the fabrication process and exact geometry of the nanostructures can be found in the Methods section.

The final nanostructure consists of three parts (Figure 1c): a magnetic thin film on the plane of the substrate, which acts as a source of magnetic DWs, a 3D NW designed to transport these DWs, and a 2D-3D interconnect region which facilitates their transmission between both. Figure 1d,e shows, respectively, scanning electron microscope (SEM) micrographs of the main structure studied in this work and the corresponding interconnect area. Figure if shows an extra 3D structure, in which the NW is disconnected from the film source via shadowing from a nanobridge fabricated before evaporation. This structure acts as a control during magnetic experiments, as detailed later.

Magneto-optical Detection. Magnetic characterization of $3 \mathrm{D}$ nanostructures is currently under intense investigation ${ }^{21,25}$ and presents many challenges. A three-dimensional geometry hinders the use of standard magnetometry or magnetic microscopy methods, suitable for planar nanostructures. We recently reported detection of suspended NWs using magnetooptical Kerr effect (MOKE). ${ }^{26}$ However, the 3D nanostructures studied here do not have any lateral separation between NW and film, which demands an approach for detection of both parts independently; this is a requirement to determine their magnetic switching mechanisms. In this work, we have developed dark-field MOKE, exploiting the different angles formed by the two components of the $3 \mathrm{D}$ nanostructure (thin film and NW) with respect to the laser beam. Light is collected with two point detectors positioned at two different angles, those result of specular reflections from either of the two areas. With this optical arrangement, we detect the magnetic switching of a single 3D NW and the film around it, independently and simultaneously (see Figure 2a). This overcomes detection limits of a standard MOKE configuration, where Kerr signal is completely dominated by the thin film (Supporting Information). Magneto-optical detection at different angles has been employed previously in transmission configuration for films with varying refractive index ${ }^{27}$ and in diffraction-MOKE of nanoelement arrays; ${ }^{28}$ here, individual 3D nanostructures are detected by using their geometry to distinguish parts which are not separated laterally but lie at different angles to the incident laser. The experimental configuration includes three sets of coils, making it possible to perform complex magnetic field sequences in 3D. A complete description of the optical setup can be found in the Supporting Information.

The successful implementation of dark-field MOKE is illustrated in Figure $2 b-e$. Figure $2 b, c$ shows optical images of the sample collected with a CCD camera positioned at the angle corresponding to the black detector shown in Figure 2a (i.e., only laser reflections at that angle-corresponding to the NW-are observed). In Figure 2b, the laser spot is positioned on top of a 3D NW (black dots), resulting in an image with a well-defined laser reflection. This is contrary to Figure 2c, where the laser beam is placed $20 \mu \mathrm{m}$ away from the NW (dashed circle), leading to no laser reflection. Additionally, Figure 2 d,e shows hysteresis loops measured by the blue and black detectors upon application of magnetic fields. The magnetic switching of NW and thin film occur at very distinct field values, with no observable cross-contamination between the two measurements.

Domain Wall Conduit Behavior in 3D Nanowires. The switching mechanisms of 3D NWs under external magnetic fields have been investigated using dark-field MOKE. The complex geometry of the nanostructures makes it challenging to interpret isolated magnetic hysteresis loops, as any applied field has a different projection along different parts of the nanostructure. In order to aid interpretation, identical experiments have been carried out for the disconnected and interconnected nanostructures of Figure 1d,f. Figure 3 summarizes this study, including the types of magnetic fields employed (Figure 3a), as well as hysteresis loops (Figure 3b,c) and switching field diagrams (Figure 3d,e) for the two nanostructures, respectively. Additionally, Figure $3 \mathrm{f}-\mathrm{i}$ shows results from micromagnetic simulations in an interconnected $3 \mathrm{D}$ system such as the one investigated experimentally, for comparison with Figure 3e.

Magnetic fields consist of a low-frequency oscillating $x$-field $\left(\mathrm{H}_{x}\right)$ along the NW axis, combined with constant $z$-offsets $\left(\mathrm{H}_{z}\right)$ normal to it (along its thickness direction) (Figure 3a). Such fields have the same positive and negative projections along any element that lies in the $x y$ plane. Conversely, those projections differ for any element not lying in that plane. This is observed for the disconnected structure in Figure 3b: NW MOKE loops are symmetric as a function of $\mathrm{H}_{x}$ for any $\mathrm{H}_{z}$ but become shifted (geometrically biased) for the film when a finite $\mathrm{H}_{z}$ is applied. The differing symmetry of both components, including the geometrical bias of the film, is readily observed in a $\mathrm{H}_{x}-\mathrm{H}_{z}$ switching diagram (Figure $3 \mathrm{~d}$ ). Here, red and turquoise dots correspond to the switching field values for NW and film, respectively. For such a disconnected system, both NW and film switch via the nucleation of domains, independently from each other. The resulting switching lines are therefore perpendicular to the plane of each component: vertical lines for the NW $\left(\mu_{0} \mathrm{H}_{\mathrm{N}} \simeq 5.5 \mathrm{mT}\right.$ nucleation field for all $\left.\mathrm{H}_{z}\right)$ and slanted (very close to each other $- \pm 0.2 \mathrm{mT}$ coercivity for $\mathrm{H}_{z}=$ 0 ) lines for the film (see the inset of Figure $3 \mathrm{~d}$ for axes and normal directions of both). As expected, the film switching occurs at much lower values than for the NW, due to the magnetic softness of Permalloy and the lack of lateral confinement in the film. The negligible $\mathrm{H}_{z}$ dependence observed for NW nucleation lines is also consequence of the small $\mathrm{H}_{z}$ field values applied in comparison to its demagnetizing field along the $z$-direction and is consistent with nucleation via curling of a small oblate ellipsoid (see Methods).

In the case of the interconnected nanostructure (Figure $3 \mathrm{c}, \mathrm{e})$, the magnetic behavior of the film is the same as before, whereas the NW now presents a richer switching landscape. This includes asymmetric MOKE loops (Figure 3c) and a switching diagram formed by four main types of field events (Figure $3 \mathrm{e}$ ). For large $\mathrm{H}_{z}$ offsets, the situation is analogous to previous studies in 2D L-shaped NWs under equivalent field routines, ${ }^{29}$ presenting very asymmetric hysteresis loops (see $\mu_{0} \mathrm{H}_{z}=+11.3 \mathrm{mT}$ in Figure $3 \mathrm{c}$ ). This is a consequence of the large film geometrical bias, which prevents the film from switching while the NW does. Two NW reversal mechanisms thus exist; either the propagation of a DW located between film and NW when the film's magnetization opposes the NW's, or, alternatively, the nucleation of new domains within the NW when no DW is present. ${ }^{29}$ The relative sign of $\mathrm{H}_{x}$ and $\mathrm{H}_{z}$ 


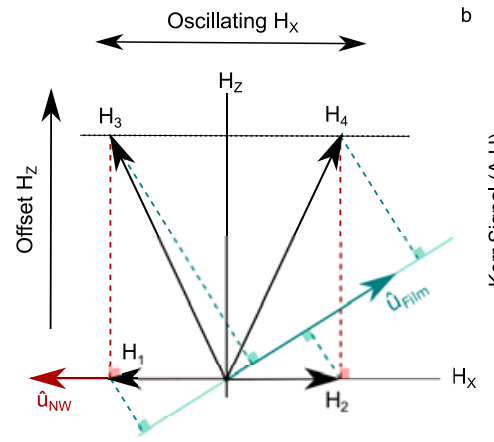

b

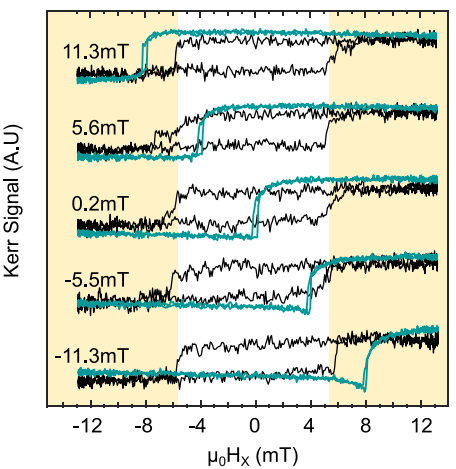

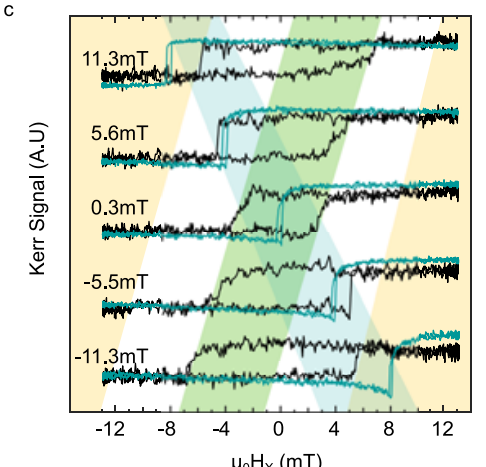

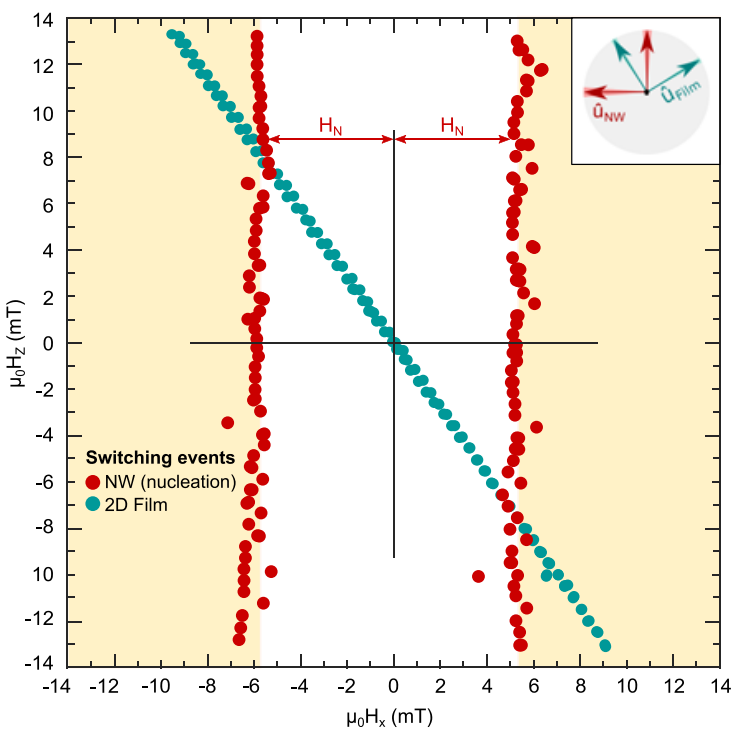

e
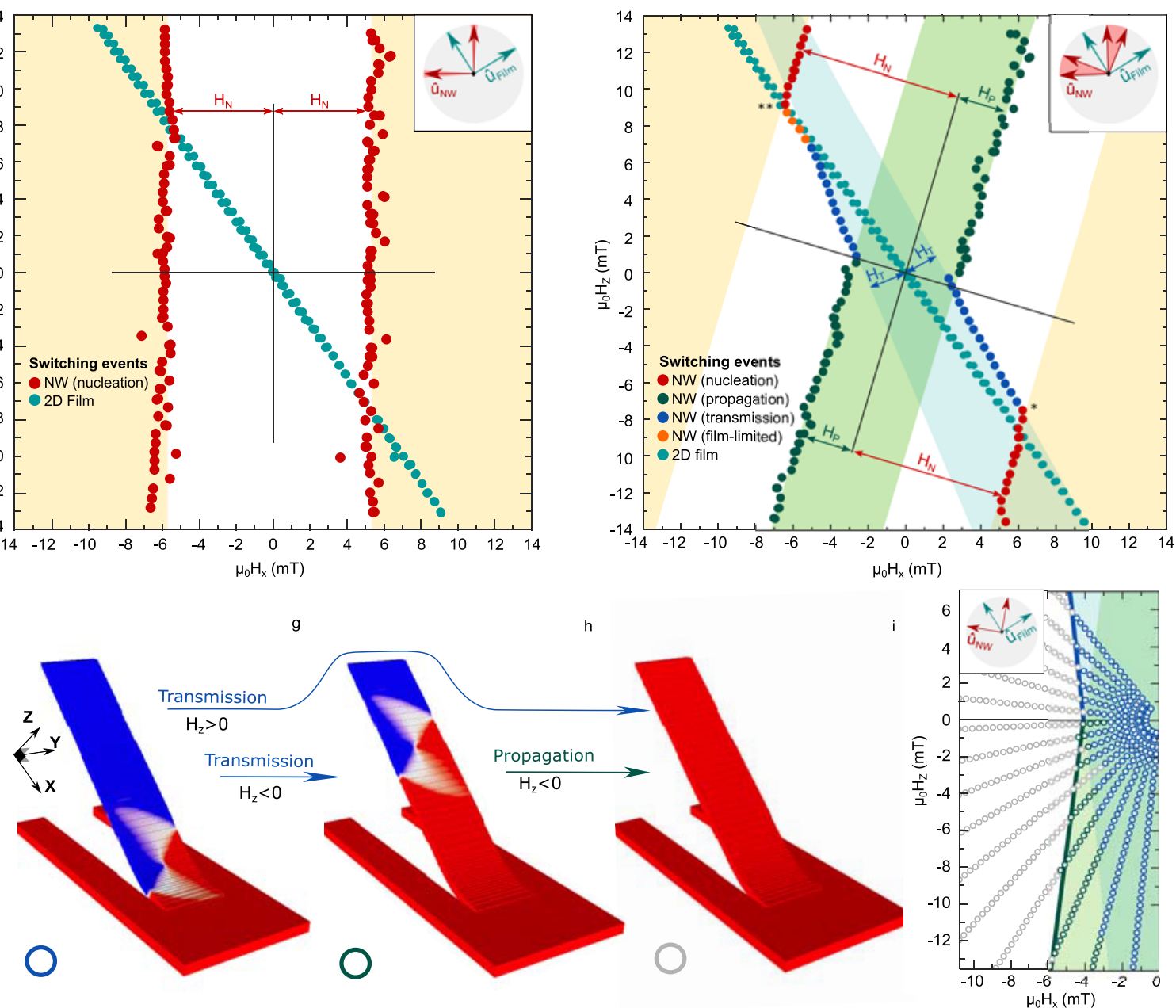

Figure 3. Magnetic characterization. (a) Effect of an offset field $\left(\mathrm{H}_{z}\right)$ on the projections of an oscillatory field $\left(\mathrm{H}_{x}\right)$ onto the NW and film. All projections are symmetric about $\mathrm{H}_{x}=0$ except for $\mathrm{H}_{3}$ and $\mathrm{H}_{4}$ onto the film. (b,c) Subset of hysteresis loops at different $\mathrm{H}_{z}$ offsets, for disconnected (b) and connected (c) NWs. Turquoise (black) lines corresponds to 2D film (3D NW) signals detected simultaneously using dark-field MOKE. Shading as in (d,e) diagrams guides the eye. (d) $\left(\mathrm{H}_{x}, \mathrm{H}_{z}\right)$ diagram of switching fields for a disconnected NW and underlying 2D film. Switching fields align perpendicularly to their corresponding structure. Areas where the NW has (not) switched are indicated with light orange (white). (e) $\left(\mathrm{H}_{x}, \mathrm{H}_{z}\right)$ diagram of switching fields for a NW interconnected to a $2 \mathrm{D}$ film. NW points are color separated depending on the switching process. The range of fields for which transmission (propagation) is not possible are shaded light blue (green). Fields for which nucleation is possible are shaded light orange. $(f-i)$ Micromagnetic simulations investigating the depinning of vortex walls from the interconnect area under fields applied at different angles. Snapshots of the simulations include domain wall pinned at interconnect (f), at the NW (g), and the fully switched system (h). The two possible NW switching processes are indicated for $\mathrm{H}_{z}>\mathbf{0}$ (single step: transmission) and $\mathbf{H}_{z}<\mathbf{0}$ (two steps: transmission + propagation). (i) Map of magnetic states shown in $\mathrm{f}$ (blue), g (green), $\mathrm{h}$ (gray). Green and blue lines correspond to switching via propagation (two-steps) and transmission (one step), respectively. Color shading as in (e) to guide the eye. Insets $(d, e, i)$ : Directions for nanowire and film axes, and their normal vectors, as determined from SEM images. Angular range where nucleation and propagation can take place is indicated by light red shading. 

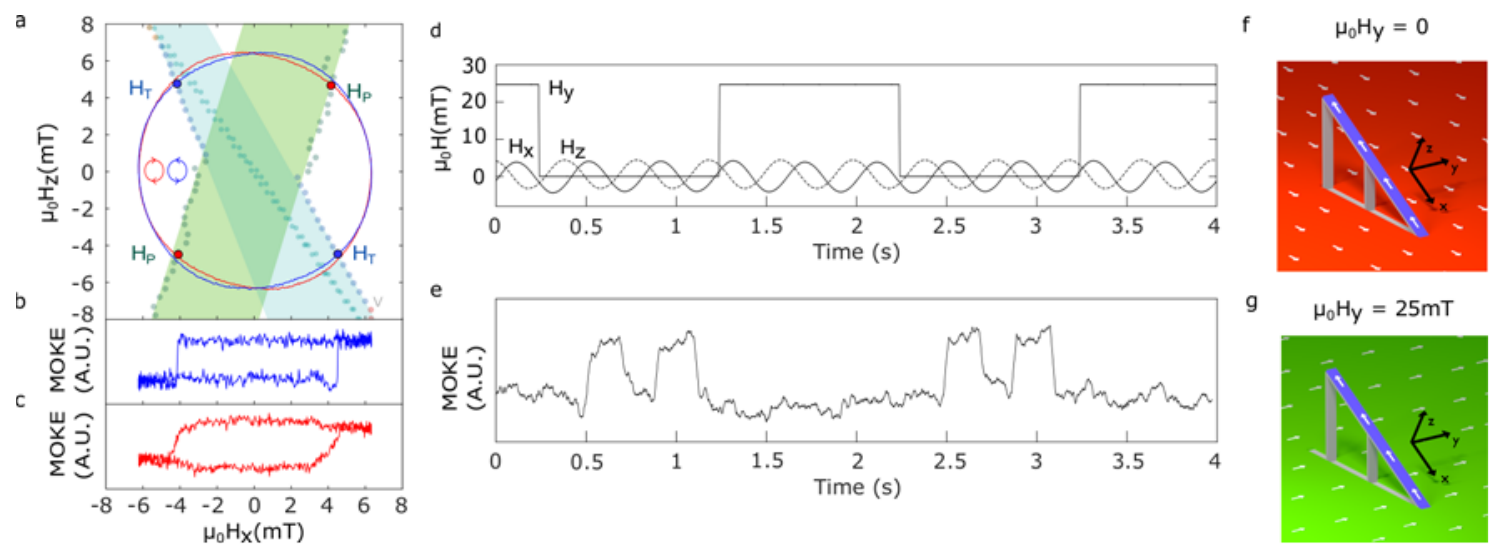

Figure 4. System operation. $(a-c)$ Control over switching by application of rotating magnetic fields. (a) Fields applied are plotted as red and blue lines, with corresponding MOKE signals below $(b, c)$. Points where switching occurs for each field sequence are marked by red and blue dots correspondingly. The diagram of Figure $3 \mathrm{~d}$ is superimposed for reference. (d) Magnetic fields applied as a function of time. A transverse field $\left(\mathbf{H}_{y}\right)$ is employed as a magnetic gate to control the injection of domains from the film, which are transmitted using rotating $\left(\mathbf{H}_{x}, \mathbf{H}_{z}\right)$ magnetic fields. (e) Corresponding time-dependent MOKE signal. $(\mathrm{f}, \mathrm{g})$ Schematic of the magnetic configuration of the system before NW switching without (f) and with $\mathrm{Hy}(\mathrm{g})$.

determines whether or not a DW is present between NW and film, fixing the reversal mechanism. In this NW, switching via nucleation is substantially sharper than that via propagation. Since both types of events occur within the NW plane, straight lines with equal slope are observed (red and green dots in Figure $3 \mathrm{e}$ ). However, they are not perfectly perpendicular to the $x$-direction, as for nucleation in the disconnected NW (Figure 3d), but have a lower slope. We associate this deviation to geometrical waviness in the connected $\mathrm{NW}$, as observed by SEM (Supporting Information). The range of possible axes for the NW inferred from SEM measurements is indicated in the inset of Figure $3 \mathrm{~d}$, with both nucleation and propagation lines falling within this angular range. Taking this into account, nucleation lines fit well to the curling model, ${ }^{30}$ with same nucleation volume as the disconnected NW (Methods). Moreover, we can define values for nucleation and propagation fields $\left(\mathrm{H}_{\mathrm{N}}\right.$ and $\mathrm{H}_{\mathrm{P}}$, respectively), by measuring the distance from either switching line to a parallel which crosses the origin, as indicated in the diagram. We obtain $\mu_{0} \mathrm{H}_{\mathrm{N}}=8.4 \mathrm{mT}$ and $\mu_{0} \mathrm{H}_{\mathrm{P}}=2.8 \mathrm{mT}$.

For smaller $\mathrm{H}_{z}$, a different regime is accessed as the film now switches before the NW and acts as a source of DWs for both positive and negative $\mathrm{H}_{x}$. A noticeable asymmetry is still observed in the hysteresis loops for this regime (see $\mu_{0} \mathrm{H}_{z}=$ $+5.6 \mathrm{mT}$ in Figure 3c). Green and blue dots in the diagram of Figure $3 \mathrm{e}$ represent the two (positive and negative) switching fields. From the different slopes and sharpness, we infer that the green switching is in fact the continuation of propagation events, now for smaller $\mathrm{H}_{z}$ offsets. The inclination of the blue lines indicates, however, switching within a different plane, located between film and NW. Following geometrical arguments, this corresponds to the interconnect between both (Figure 1d), as detailed later. We thus associate a transmission field $\left(\mathrm{H}_{\mathrm{T}}\right)$ to this type of switching, which characterizes DW depinning at the interconnect area, with $\mathrm{H}_{\mathrm{T}}=2.0 \mathrm{mT}$ defined analogously to $\mathrm{H}_{\mathrm{N}}$ and $\mathrm{H}_{\mathrm{P}}$ (see Figure $3 \mathrm{e}$ ). The dependence observed for both propagation and transmission lines fits well with the Kondorsky model ${ }^{31}$ for DW motion (Methods). Remarkably, with $\mathrm{H}_{\mathrm{N}}>\mathrm{H}_{\mathrm{P}}$ and $\mathrm{H}_{\mathrm{T}}$, the injection of DWs from the film into the 3D NW is realized, with the system operating as an effective $3 \mathrm{D}$ conduit of DWs.
In order to understand in detail the mechanisms for DW motion after injection from the film, we have performed micromagnetic simulations ${ }^{32}$ (Methods). For this, we have investigated the depinning of vortex DWs at the interconnect area (Figure $3 \mathrm{f}-\mathrm{i}$ ) by applying $\mathrm{H}_{x}-\mathrm{H}_{z}$ fields from 0 to $15 \mathrm{mT}$ at different angles. The $3 \mathrm{D}$ geometry numerically investigated mimics the key features observed in the experimental nanostructures, as observed by SEM. This includes a small narrowing in the NW geometry at two locations: one at the start of the interconnect and a second at an intermediate location within the NW, $600 \mathrm{~nm}$ above its base. These two defects are expected to act as DW pinning areas. ${ }^{13}$ Figure $3 \mathrm{i}$ shows a diagram generated from the simulations, where each point corresponds to one simulation step. Three different colors denote the different magnetic states observed, as shown in Figure $3 \mathrm{f}-\mathrm{h}$. DW pinning at the interconnect area (Figure $3 \mathrm{f}$ ) and at the intermediate defect (Figure $3 \mathrm{~g}$ ) are represented as blue and green open circles, respectively, and a completely reversed NW (Figure 3h) as gray open circles. The values and symmetry obtained for transmission and propagation lines in Figure 3i (green and blue lines) are in good agreement with experiments. Simulations show how the relative sign of $\mathrm{H}_{x}$ and $\mathrm{H}_{z}$, and therefore the differing projections of the total field over the NW and interconnect, determines whether the NW switching is dominated by either transmission or propagation. For positive $\mathrm{H}_{z}$, the NW switches in a single step (from Figure $3 \mathrm{f}$ to $3 \mathrm{~h}$ ): the DW is transmitted from the interconnect and is not pinned at the intermediate defect, due to the large projection of the field over the NW. This is contrary to negative $\mathrm{H}_{z}$ cases, with a small field projection. Here, the DW is first depinned from the interconnect and gets trapped at the intermediate defect (Figure $3 \mathrm{~g}$ ), until DW propagation takes place, leading to full switching (Figure $3 \mathrm{~h}$ ). A video and further illustrations of the different switching mechanisms are included in the Supporting Information.

In addition to the geometrical depinning effect ${ }^{33}$ reproduced by simulations, experiments (Figure 3e) show a slightly different slope for the two transmission branches. This creates a significant asymmetry in the diagram at the areas marked * and **; whereas * denotes a direct crossover between transmission and nucleation, ** switching fields deviate 
(orange points) from transmission, to follow instead the switching line of the film, before the crossover with nucleation. This is a direct consequence of the film acting as a source of domains for the NW at this region of the diagram: DW injection is film-limited. The different slopes for the transmission lines can be understood in terms of DW chiralitydependent depinning, ${ }^{28,34}$ with $\mathrm{H}_{z}$ chirality selection due to the interconnect curvature, as previously reported for Permalloy 3D curved elements. ${ }^{35}$

3D Conduit Operation. The above results demonstrate a good DW conduit behavior for the interconnected 3D NWs under investigation. They also show how DWs injected from the $2 \mathrm{D}$ film get trapped at different areas of the conduit, depending on the magnitude and direction of the applied field with respect to the three elements of the system (film, interconnect, and NW). This has been exploited for advanced field-driven operation of the nanodevice, as shown in Figure 4a. The figure shows an experiment where $\mathrm{H}_{x}-\mathrm{H}_{z}$ rotating magnetic fields (red and blue lines) have been applied, overlaid onto the diagram of Figure 3e. These fields result in symmetric hysteresis loops, with the NW switching either sharply via DW transmission (Figure 4b) if the field is counterclockwise (CCW) or progressively via propagation (Figure 4c) if it is clockwise $(\mathrm{CW})$. The sense of rotation of the field determines the order in which transmission and propagation lines are crossed, allowing us to control if the DW injected from the film gets trapped, or not, at defects along the NW. This property could be exploited to select different functionalities of the 3D nanomagnetic conduit: ${ }^{21}$ acting either as a magnetic $3 \mathrm{D}$ interconnector when no trapping is produced within the NW, linking planes hosting functional DW devices; or as a 3D fielddriven racetrack memory component, with NW width modulation utilized to trap DWs along its length.

Finally, we have employed fully three-dimensional magnetic fields, by combining a rotating $\mathrm{H}_{x}-\mathrm{H}_{z}$ with $\mathrm{H}_{y}$ offsets (Figure 4d). As before, under moderate $\mathrm{H}_{x}-\mathrm{H}_{z}$ CCW rotating magnetic fields and $\mathrm{H}_{y}=0$, the NW switches back and forth via the injection of domains from the film (Figure $4 \mathrm{f}$ ), which is transmitted through the interconnect. This results in an alternating NW MOKE signal as a function of time (Figure $4 \mathrm{e})$. On the contrary, the addition of $\mathrm{H}_{y}$ magnetically saturates the film (Figure $4 \mathrm{~g}$ ), inhibiting the injection of DWs. As the applied rotating field is not enough to nucleate domains in the NW, no switching is produced, and a constant low MOKE signal is obtained in this case (Figure 4e). This result encapsulates the full vectorial nature of the $3 \mathrm{D}$ system, where the application of $\mathrm{a} \mathrm{H}_{y}$ offset acts as a magnetic gate, controlling the injection of DWs from the $2 \mathrm{D}$ film into the $3 \mathrm{D} \mathrm{NW}$, and exemplifies how the full $x y z$ magnetic energy landscape can be exploited in 3D nanomagnetic systems.

\section{CONCLUSIONS}

We have demonstrated an advanced platform for the fabrication and study of three-dimensional magnetic nanostructures. Highquality magnetic material has been successfully shaped in three dimensions by thermal evaporation onto a nonmagnetic scaffold fabricated using 3D nanoprinting. Different areas of the system can be detected independently using a dark-field magneto-optical method tailored to the $3 \mathrm{D}$ geometry of the nanostructure. A nanomagnetic conduit which can transport magnetic information in three dimensions has been demonstrated, and the mechanisms driving such a process have been characterized. The methods demonstrated in this work can potentially be extended to complex 3D geometries and nanostructured multilayered materials. These developments may allow spintronic devices that can take advantage of the three dimensions, increasing functionality and density. In particular, the techniques presented here can be potentially extended to NWs with different cross sections for the experimental investigation of dynamic effects recently predicted in 3D nanowires. ${ }^{21}$ Many possibilities also exist in fields of study such as magnetic spin-ice ${ }^{7}$ and magnetic neural networks, ${ }^{36}$ in which a great degree of connectivity between elements would be allowed. Furthermore, the applicability of the techniques demonstrated is extensible to any nanotechnology area which may benefit from extending into the third dimension.

\section{METHODS}

The nonmagnetic scaffold was fabricated in a SEM/FIB microscope using trimethyl(methylcyclopentadienyl)platinum as a gas precursor. Growth conditions: $30 \mathrm{kV}$ acceleration voltage, $25 \mathrm{pA}$ nominal beam current, base pressure of $2 \times 10^{-6} \mathrm{mbar}$, and growth pressure of $7 \times$ $10^{-6}$ mbar. Stream files specifying substrate coordinates and dwell time were employed at a magnification of $35000 \times$. The growth is performed with a substrate tilt of $30^{\circ}$ in the direction of the ramps. The angle formed by the ramp with the substrate is controlled by scanning the electron beam at a constant speed $v_{s}{ }^{35}$ Slope, length, and width of the NWs studied here were set to $30^{\circ}, 5 \mu \mathrm{m}$, and $300 \mathrm{~nm}$, respectively, values designed for compatibility with magneto-optical detection. First, two support pillars of $300 \mathrm{~nm} \times 60 \mathrm{~nm}$ cross section and heights of 3 and $2.2 \mu \mathrm{m}$ are deposited by scanning the beam in a single pixel line with a $20 \mathrm{~nm}$ pitch. Then the ramp is grown by scanning the beam in a serpentine pattern with longitudinal pitch of 1 $\mathrm{nm}$ and transverse pitch of $20 \mathrm{~nm}$. The dwell time is increased from 0 to $20 \mathrm{~ms}$ over the first $500 \mathrm{~nm}$ of patterning, keeping it constant for the rest of the deposit. This generates a high-quality substrate-toscaffold transition, which is transferred to the magnetic thin film upon thermal evaporation. The shadowing nanobridge of the control structure was grown as the intersection of two shorter ramps using the same conditions. A $50 \mathrm{~nm}$ Permalloy thin film was thermally evaporated perpendicularly to the $2 \mathrm{D}$ substrate, at a rate of $1.7 \mathrm{~nm} /$ min and growth pressure of $4.8 \times 10^{-6} \mathrm{mbar}$. Chamber base pressure: $2.5 \times 10^{-7}$ mbar. After evaporation, NWs showed small roughness, with slope deviations from their mean value of up to $10^{\circ}$.

The resulting 3D magnetic NWs have rectangular cross sections (300 nm wide, $50 \mathrm{~nm}$ thick), the same as conventional planar NWs patterned by standard lithography methods, where width $>$ thickness. We therefore expect the same type of DW structures, static and dynamic behaviors as in previous works where $2 \mathrm{D}$ NWs have been investigated. ${ }^{38,39}$ In this case, the most stable configuration corresponds to a vortex DW. ${ }^{37}$ Additional factors especially relevant in 3D NWs that may affect DW dynamics are defects such as NW roughness ${ }^{39,40}$ and the application of magnetic fields perpendicularly to the NW axis. $^{41}$

For magneto-optical characterization, we employ a p-polarized laser beam, which is incident at $45^{\circ}$ to the plane of the NW ( $x y$ plane) and $12^{\circ}$ to the plane of the substrate. The setup is aligned so that NW, laser, and normal to the substrate lie in the $x z$ plane, resulting in two reflected beams, also in the $x z$ plane. These two beams correspond to the reflections from the NW and the substrate and are picked up by the black and blue detectors, respectively (see Figure 2a). A magnetic quadrupole located in the $x y$ plane is used to apply $\mathrm{H}_{x}$ and $\mathrm{H}_{y}$ fields of up to $30 \mathrm{mT}$, and a single pole coil can be used to apply $\mathrm{H}_{z}$ fields of up to $15 \mathrm{mT}$. Three lenses (not shown) are used to focus the laser spot on the substrate (full width at half-maximum $\approx 5 \mu \mathrm{m}$ ) and collect the two reflected beams. Changes in MOKE ellipticity are measured using a combination of $\lambda / 4$ plate and analyzer for each reflected beam. Oscillatory $\mathrm{H}_{x}$ fields at $3 \mathrm{~Hz}$ are used to modulate the magnetic signal. $^{42}$ The biggest sources of uncertainty in the measurements are the angle between the substrate and the $x y$ plane, which was set to 33 
$\pm 3^{\circ}$ to better match NW angles obtained experimentally (see Supporting Information), and the distance between substrate and $z$ coil, which introduces a $10 \%$ uncertainty for $\mathrm{H}_{z}$. Nanostructure and film are independently detected with SNR of 3.6 and 12, respectively. Switching events in the diagrams are defined as a sign change of MOKE signal. Supporting Information shows a schematic of the complete optical setup; grayed out components are not used. Lamp is turned on only during alignment.

Domain wall nucleation within magnetic NWs was fitted to the curling model for an oblate ellipsoid with minor and major semiaxes $a$ and $b$, respectively, in which the nucleation field is given by ${ }^{29}$

$$
\mu_{0} H_{N}(a, b, \Omega)=\frac{\mu_{0} M_{S}\left(2 D_{a}(a, b)-\frac{k}{S(a)^{2}}\right)\left(2 D_{b}(a, b)-\frac{k}{S(a)^{2}}\right)}{2 \sqrt{\left(2 D_{a}(a, b)-\frac{k}{(S(a))^{2}}\right)^{2} \sin ^{2}(\Omega)+\left(2 D_{b}(a, b)-\frac{k}{(S(a))^{2}}\right)^{2} \cos ^{2}(\Omega)}}
$$

where $\Omega$ is the angle between the applied field and the minor semiaxis of the ellipsoid, $M_{s}(\mathrm{~A} / \mathrm{m})$ is the saturation magnetization, $D_{a}(a, b)$ and $D_{b}(a, b)$ are the demagnetizing factors of the ellipsoid along its minor and major axes, respectively, $k$ is a geometrical parameter between 1.3793 and $1.424,{ }^{36}$ and $S(a)=a / R_{0}$ with

$$
R_{0}=\sqrt{\frac{2 \pi A_{\mathrm{ex}}}{\mu_{0} M_{S}^{2}}}
$$

where $A_{\text {ex }}(\mathrm{J} / \mathrm{m})$ is the exchange stiffness. Defining $m=a / b$, the demagnetizing factors $D_{a}(a, b)$ and $D_{b}(a, b)$ are given by ${ }^{37}$

$$
D_{a}=\frac{1}{1-m^{2}}\left(1-\frac{m \cos ^{-1}(m)}{\sqrt{1-m^{2}}}\right) ; \quad D_{b}=\frac{1}{2}\left(1-D_{a}\right)
$$

We fit the nucleation volume assuming an oblate ellipsoid, which is exactly contained within the thickness of the NW, that is, $2 a=$ $\cos \left(30^{\circ}\right) \times 50 \mathrm{~nm}$, where $50 \mathrm{~nm}$ is the thickness of Permalloy deposited perpendicularly to the $2 \mathrm{D}$ film and $30^{\circ}$ is the nominal angle between the NW and the 2D film; $M_{\mathrm{S}}=8 \times 10^{5} \mathrm{~A} / \mathrm{m}$ and $A_{\mathrm{ex}}=1.3 \times$ $10^{-11} \mathrm{~J} / \mathrm{m}$. As described in the main text, nucleation fields at $\Omega=90^{\circ}$ were measured as the distance between nucleation lines in Figure 3d,e and a parallel passing through the origin; $\mu_{0} \mathrm{H}_{\mathrm{N}}=5.7 \pm 0.4 \mathrm{mT}$ and $\mu_{0} \mathrm{H}_{\mathrm{N}}=8.4 \pm 0.1 \mathrm{mT}$ for disconnected and connected NWs, obtaining estimations for $b$ of 80 and $78 \mathrm{~nm}$, respectively.

The angular dependence of Kondorsky-type domain wall propagation is given by $^{37}$

$$
\mathrm{H}_{\mathrm{sw}}(\theta)=\frac{\mathrm{H}_{0}}{\cos (\theta)}
$$

where $\theta$ is the angle of the applied field. This relationship is equivalent to straight lines in an $\mathrm{H}_{z}-\mathrm{H}_{x}$ diagram. For both propagation and transmission lines, $\mathrm{H}_{0}$ values were determined by measuring the distance between each relevant line and a parallel passing through the origin, as explained in the main text.

Micromagnetic simulations were performed using version 3.9 of the software Mumax $3^{31}$ on an Nvidia GTX1070 graphics card. A cell size of $5 \mathrm{~nm}$ was chosen, and a total volume of $512 \times 256 \times 128$ cells $(2.56$ $\mu \mathrm{m} \times 1.28 \mu \mathrm{m} \times 0.64 \mu \mathrm{m}$ ) was simulated. The NW slope was set to $40^{\circ}$ with respect to the $2 \mathrm{D}$ film. The thickness of the structure is 50 $\mathrm{nm}$ perpendicularly to the $2 \mathrm{D}$ film to emulate the Permalloy evaporation process. Exchange stiffness is $A_{\mathrm{ex}}=13 \times 10^{-12} \mathrm{~J} / \mathrm{m}$, and saturation magnetization is $M_{\mathrm{s}}=8 \times 10^{5} \mathrm{~A} / \mathrm{m}$. Two constrictions decreasing the width of the ramp by $5 \mathrm{~nm}$ at each side ( 1 cell) were included in the ramp and the interconnect (see Supporting Information) as determined by SEM micrographs. When reaching the $2 \mathrm{D}$ film, the lower constriction penetrates $20 \mathrm{~nm}$ into it.

The system was initialized with a random circular patch $250 \mathrm{~nm}$ in radius at the base of the interconnect. The magnetization was then relaxed using the built in $\operatorname{relax}()$ function of Mumax 3 obtaining a vortex domain wall (Figure 3f). The stray fields from a larger 2D film and ramp were imported at the boundaries of the system to avoid the formation of closure domains. The relaxed configuration was stored and used to initialize all field sequences.

Field sequences from 0 to $15 \mathrm{mT}$ were applied at different angles in 40 steps, performing a steepest gradient minimization after each increase using the built-in function minimize() in Mumax3. The minimizer stop parameter was set to $10^{-6}$.

The maximum angle between spins remained below $43^{\circ}$ throughout the whole simulation, corresponding to a maximum error of $\simeq 4 \%$ in exchange energy. ${ }^{31}$ The video in Supporting Information shows the evolution for two field sequences at positive and negative $\mathrm{H}_{z}$ offsets. The complete script used to perform the simulations is available in the Supporting Information.

\section{ASSOCIATED CONTENT}

\section{Supporting Information}

The Supporting Information is available free of charge on the ACS Publications website at DOI: 10.1021/acsnano.7b05105.

Experimental and technical details (PDF)

Video illustrating the two modes of domain wall conduction (AVI)

\section{AUTHOR INFORMATION}

\section{Corresponding Author}

*E-mail: af457@cam.ac.uk.

ORCID 1

Amalio Fernández-Pacheco: 0000-0002-3862-8472

Notes

The authors declare no competing financial interest.

\section{ACKNOWLEDGMENTS}

This research is funded by an EPSRC Early Career Fellowship EP/M008517/1, a Winton Fellowship, and by a European Erasmus Mobility program. D.S.H. acknowledges funding from a Girton College Pfeiffer Scholarship. J.-W. L acknowledges support from a Newton International Fellowship scheme NF150217. We thank R.P. Cowburn and M. Sanz-Hernández for fruitful discussions.

\section{REFERENCES}

(1) Allwood, D. A.; Xiong, G.; Faulkner, C. C.; Atkinson, D.; Petit, D.; Cowburn, R. P. Magnetic Domain-Wall Logic. Science 2005, 309, $1688-1692$.

(2) Parkin, S. S. P.; Hayashi, M.; Thomas, L. Magnetic Domain-Wall Racetrack Memory. Science 2008, 320, 190-194.

(3) Parkin, S.; Yang, S.-H. Memory on the Racetrack. Nat. Nanotechnol. 2015, 10, 195-198.

(4) Woo, S.; Delaney, T.; Beach, G. S. D. Magnetic Domain Wall Depinning Assisted by Spin Wave Bursts. Nat. Phys. 2017, 13, 448454.

(5) Sohn, H.; Nowakowski, M. E.; Liang, C.; Hockel, J. L.; Wetzlar, K.; Keller, S.; McLellan, B. M.; Marcus, M. A.; Doran, A.; Young, A.; Kläui, M.; Carman, G. P.; Bokor, J.; Candler, R. N. Electrically Driven Magnetic Domain Wall Rotation in Multiferroic Heterostructures to Manipulate Suspended On-Chip Magnetic Particles. ACS Nano 2015, 9, 4814-4826. 
(6) Mattheis, R.; Glathe, S.; Diegel, M.; Hübner, U. Concepts and Steps for the Realization of a New Domain Wall Based Giant Magnetoresistance Nanowire Device: From the Available 24 Multiturn Counter to a 212 Turn Counter. J. Appl. Phys. 2012, 111, 113920.

(7) Pushp, A.; Phung, T.; Rettner, C.; Hughes, B. P.; Yang, S.-H.; Thomas, L.; Parkin, S. S. P. Domain Wall Trajectory Determined by Its Fractional Topological Edge Defects. Nat. Phys. 2013, 9, 505-511.

(8) Donolato, M.; Vavassori, P.; Gobbi, M.; Deryabina, M.; Hansen, M. F.; Metlushko, V.; Ilic, B.; Cantoni, M.; Petti, D.; Brivio, S.; Bertacco, R. On-Chip Manipulation of Protein-Coated Magnetic Beads via Domain-Wall Conduits. Adv. Mater. 2010, 22, 2706-2710.

(9) Corte-León, H.; Krzysteczko, P.; Schumacher, H. W.; Manzin, A.; Cox, D.; Antonov, V.; Kazakova, O. Magnetic Bead Detection Using Domain Wall-Based Nanosensor. J. Appl. Phys. 2015, 117, $17 \mathrm{E} 313$.

(10) Ono, T.H.; Shigeto, K.; Mibu, K.; Hosoito, N.; S, T. Propagation of a Magnetic Domain Wall in a Submicrometer Magnetic Wire. Science 1999, 284, 468-470.

(11) Nakatani, Y.; Thiaville, A.; Miltat, J. Head-to-Head Domain Walls in Soft Nano-Strips: A Refined Phase Diagram. J. Magn. Magn. Mater. 2005, 290-291, 750-753.

(12) Nguyen, V. D.; Fruchart, O.; Pizzini, S.; Vogel, J.; Toussaint, J.C.; Rougemaille, N. Third Type of Domain Wall in Soft Magnetic Nanostrips. Sci. Rep. 2015, 5, 12417.

(13) Petit, D.; Jausovec, A.-V.; Read, D.; Cowburn, R. P. Domain Wall Pinning and Potential Landscapes Created by Constrictions and Protrusions in Ferromagnetic Nanowires. J. Appl. Phys. 2008, 103, 114307.

(14) Franken, J. H.; Swagten, H. J. M.; Koopmans, B. Shift Registers Based on Magnetic Domain Wall Ratchets with Perpendicular Anisotropy. Nat. Nanotechnol. 2012, 7, 499-503.

(15) Currivan-Incorvia, J. A.; Siddiqui, S.; Dutta, S.; Evarts, E. R.; Zhang, J.; Bono, D.; Ross, C. A.; Baldo, M. A. Logic Circuit Prototypes for Three-Terminal Magnetic Tunnel Junctions with Mobile Domain Walls. Nat. Commun. 2016, 7, 10275.

(16) Emori, S.; Bauer, U.; Ahn, S.-M.; Martinez, E.; Beach, G. S. D. Current-Driven Dynamics of Chiral Ferromagnetic Domain Walls. Nat. Mater. 2013, 12, 611-616.

(17) Yang, S.-H.; Ryu, K.-S.; Parkin, S. Domain-Wall Velocities of up to 750 M S (-1) Driven by Exchange-Coupling Torque in Synthetic Antiferromagnets. Nat. Nanotechnol. 2015, 10, 221-226.

(18) Jué, E.; Safeer, C. K.; Drouard, M.; Lopez, A.; Balint, P.; BudaPrejbeanu, L.; Boulle, O.; Auffret, S.; Schuhl, A.; Manchon, A.; Miron, I. M.; Gaudin, G. Chiral Damping of Magnetic Domain Walls. Nat. Mater. 2015, 15, 272-277.

(19) Yoshimura, Y.; Kim, K.-J.; Taniguchi, T.; Tono, T.; Ueda, K.; Hiramatsu, R.; Moriyama, T.; Yamada, K.; Nakatani, Y.; Ono, T. Soliton-like Magnetic Domain Wall Motion Induced by the Interfacial Dzyaloshinskii-Moriya Interaction. Nat. Phys. 2015, 12, 157-161.

(20) Andreas, C.; Kákay, A.; Hertel, R. Multiscale and Multimodel Simulation of Bloch-Point Dynamics. Phys. Rev. B: Condens. Matter Mater. Phys. 2014, 89, 134403.

(21) Fernández-Pacheco, A.; Streubel, R.; Fruchart, O.; Hertel, R.; Fischer, P.; Cowburn, R. P. Three Dimensional Nanomagnetism. Nat. Commun. 2017, 8, 15756.

(22) Ivanov, Y. P.; Chuvilin, A.; Lopatin, S.; Kosel, J. Modulated Magnetic Nanowires for Controlling Domain Wall Motion: Toward 3D Magnetic Memories. ACS Nano 2016, 10, 5326.

(23) Da Col, S.; Jamet, S.; Staňo, M.; Trapp, B.; Le Denmat, S.; Cagnon, L.; Toussaint, J. C.; Fruchart, O. Nucleation, Imaging, and Motion of Magnetic Domain Walls in Cylindrical Nanowires. Appl. Phys. Lett. 2016, 109, 062406.

(24) Utke, I.; Hoffmann, P.; Melngailis, J. Gas-Assisted Focused Electron Beam and Ion Beam Processing and Fabrication. J. Vac. Sci. Technol. B Microelectron. Nanom. Struct. 2008, 26, 1197.

(25) Streubel, R.; Fischer, P.; Kronast, F.; Kravchuk, V. P.; Sheka, D. D.; Gaididei, Y.; Schmidt, O. G.; Makarov, D. Magnetism in Curved Geometries. J. Phys. D: Appl. Phys. 2016, 49, 363001.

(26) Fernández-Pacheco, A.; Serrano-Ramón, L.; Michalik, J. M.; Ibarra, M. R.; De Teresa, J. M.; O’Brien, L.; Petit, D.; Lee, J.; Cowburn,
R. P. Three Dimensional Magnetic Nanowires Grown by Focused Electron-Beam Induced Deposition. Sci. Rep. 2013, 3, 1492.

(27) Patek, K.; Thiaville, A.; Miltat, J. Horizontal Bloch Lines and Anisotropic-Dark-Field Observations. Phys. Rev. B: Condens. Matter Mater. Phys. 1994, 49, 6678-6688.

(28) Verduci, T.; Rufo, C.; Berger, A.; Metlushko, V.; Ilic, B.; Vavassori, P. Fourier Magnetic Imaging. Appl. Phys. Lett. 2011, 99, 092501.

(29) Allwood, D. a.; Vernier, N.; Xiong, G.; Cooke, M. D.; Atkinson, D.; Faulkner, C. C.; Cowburn, R. P. Shifted Hysteresis Loops from Magnetic Nanowires. Appl. Phys. Lett. 2002, 81, 4005.

(30) Aharoni, A. Angular Dependence of Nucleation by Curling in a Prolate Spheroid. J. Appl. Phys. 1997, 82, 1281.

(31) Kondorsky, E. On Hysteresis of Ferromagnetics. J. Phys. (Paris) 1940, 2, 161-181.

(32) Vansteenkiste, A.; Leliaert, J.; Dvornik, M.; Helsen, M.; GarciaSanchez, F.; Van Waeyenberge, B. The Design and Verification of MuMax3. AIP Adv. 2014, 4, 107133.

(33) Lewis, E. R.; Petit, D.; Thevenard, L.; Jausovec, A. V.; O’Brien, L.; Read, D. E.; Cowburn, R. P. Magnetic Domain Wall Pinning by a Curved Conduit. Appl. Phys. Lett. 2009, 95, 152505.

(34) Omari, K. A.; Hayward, T. J. Chirality-Based Vortex DomainWall Logic Gates. Phys. Rev. Appl. 2014, 2, 44001.

(35) Hertel, R. Curvature-Induced Magnetochirality. SPIN 2013, 3, 1340009.

(36) Torrejon, J.; Riou, M.; Araujo, F. A.; Tsunegi, S.; Khalsa, G.; Querlioz, D.; Bortolotti, P.; Cros, V.; Yakushiji, K.; Fukushima, A.; Kubota, H.; Yuasa, S.; Stiles, M. D.; Grollier, J. Neuromorphic Computing with Nanoscale Spintronic Oscillators. Nature 2017, 547, 428-431.

(37) Thiaville, A.; Nakatani, Y. Domain-Wall Dynamics in Nanowires and Nanostrips. Spin Dynamics in Confined Magnetic Structures III; Springer: Berlin, 2006; pp 161-205.

(38) Beach, G. S. D.; Nistor, C.; Knutson, C.; Tsoi, M.; Erskine, J. L. Dynamics of Field-Driven Domain-Wall Propagation in Ferromagnetic Nanowires. Nat. Mater. 2005, 4, 741-744.

(39) Nakatani, Y.; Thiaville, A.; Miltat, J. Faster Magnetic Walls in Rough Wires. Nat. Mater. 2003, 2, 521-523.

(40) Martinez, E.; Lopez-Diaz, L.; Torres, L.; Tristan, C.; Alejos, O. Thermal Effects in Domain Wall Motion: Micromagnetic Simulations and Analytical Model. Phys. Rev. B: Condens. Matter Mater. Phys. 2007, $75,174409$.

(41) Kunz, A.; Reiff, S. C. Fast Domain Wall Motion in Nanostripes with out-of-Plane Fields. Appl. Phys. Lett. 2008, 93, 082503.

(42) Allwood, D. a; Xiong, G.; Cooke, M. D.; Cowburn, R. P. Magneto-Optical Kerr Effect Analysis of Magnetic Nanostructures. J. Phys. D: Appl. Phys. 2003, 36, 2175-2182. 